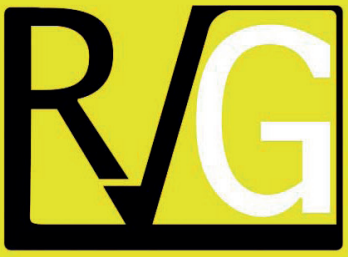

Julio - Septiembre, 2021

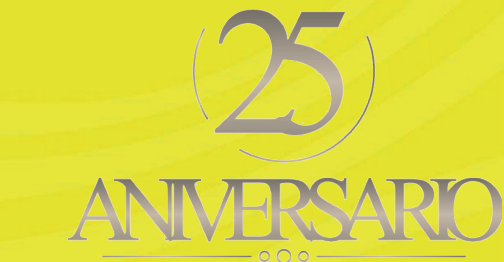

ANMERSARO
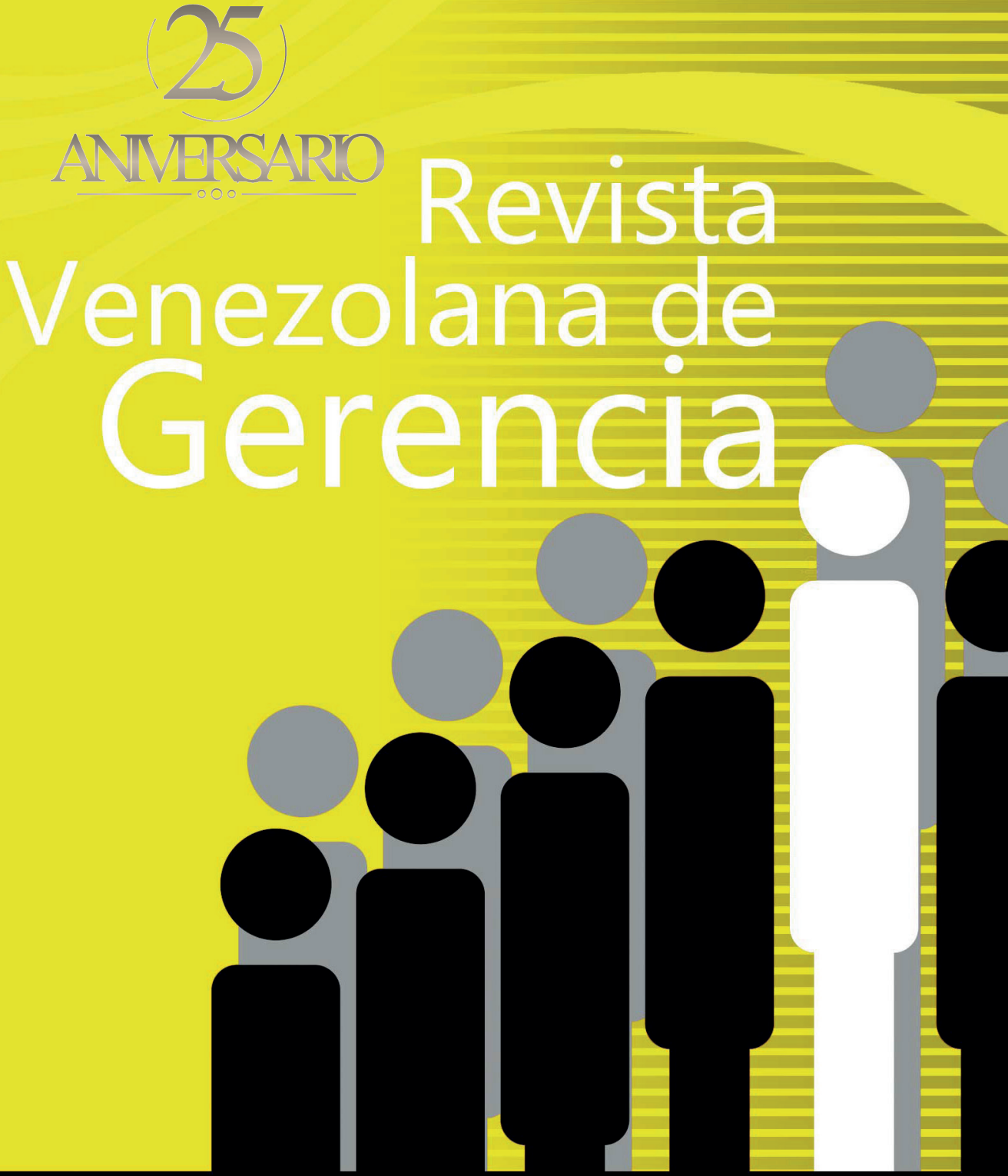

UNIVERSIDAD DEL ZULIA (LUZ)

Facultad de Ciencias Económicas y Sociales Centro de Estudios de la Empresa

ISSN 1315-99

Esta obra está bajo una licencia de Creative Comm Reconocimiento-NoComercial-Compartirlgual 3.0 Unpo http://creativecommons.org/licenses/by-nc-sa/3.0/deed.es 
COMO CITAR: Agudelo Cotes, K. J. y Bedoya Gómez, D. C. (2021). Riesgos de adaptación en franquicias: herramientas de contabilidad de gestión para mitigarlos. Revista Venezolana de Gerencia (RVG), 26(95), 832-851. https://doi. org/10.52080/rvgluz.27.95.24
Universidad del Zulia (LUZ)

Revista Venezolana de Gerencia (RVG)

Año 26 No. 95 Julio-Septiembre 2021, 832-851

ISSN 1315-9984 / e-ISSN 2477-9423

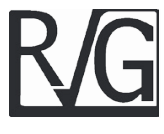

\title{
Riesgos de adaptación en franquicias: herramientas de contabilidad de gestión para mitigarlos
}

\author{
Agudelo Cotes, Karen J.* \\ Bedoya Gómez, Diana C.**
}

\section{Resumen}

El crecimiento sostenido del mercado de franquicias en países desarrollados, impulsa su afianzamiento en mercados emergentes. En Colombia se han establecido más de 500 marcas de este formato empresarial y, sin embargo, la documentación estadística o académica que describa la adaptación de este tipo de empresas en el país y sus procesos de internacionalización son escasos. Como una contribución al acervo académico y a la comprensión de este fenómeno, el presente artículo es un estudio de tres casos exploratorios que tiene como objetivo identificar los riesgos de adaptación de franquicias dedicadas a la prestación servicios de formación extracurricular para niños en Colombia. Las entrevistas fueron realizadas a los líderes responsables del proceso de expansión de la franquicia en Colombia. Los riesgos de adaptación identificados se agruparon en desarrollo comercial y animación de la red, los cuales evidencian, perfiles inadecuados en la selección de franquiciados, afectación de la marca, poco estudio de la competencia y problemas de comunicación y seguimiento.

Palabras Clave: Riesgos de adaptación en franquicias; contabilidad de gestión; internacionalización de franquicias; servicios de formación extracurricular para niños.

\section{Recibido: 10.03.21 Aceptado: 15.05.21}

Docente tiempo completo. Facultad de Ciencias Económicas y Administrativas, Departamento de Finanzas, Institución Universitaria Instituto Tecnológico Metropolitano, Medellín - Colombia. Email: karenagudelo@itm. edu.co

* Docente tiempo completo. Escuela de Administración, Programa de Contaduría Pública, Fundación Universitaria CEIPA, Sabaneta - Colombia. Email: dianabedoyag@ceipa.edu.co 


\title{
Adapting the franchise model: risks and management accounting tools to mitigate them
}

\begin{abstract}
The sustained growth of the franchise market in developed countries boosts its consolidation in emerging markets. In Colombia, more than 500 brands of this business format have settled and, however, the statistical or academic documentation that describes the adaptation of this type of companies in the country and their internationalization processes are scarce. As a contribution to the academic heritage and to the understanding of this phenomenon, this article is a study of three exploratory cases that aims to identify the risks of adaptation of franchises dedicated to providing extracurricular training services for children in Colombia. The interviews conducted to the leaders responsible of the expansion process of the franchise in Colombia. The adaptation risks identified were grouped into commercial development and network animation, which show inadequate profiles in the selection of franchisees, brand involvement, little study of the competition, and communication and monitoring problems.
\end{abstract}

Key words: Risk in adapting franchises; management accounting; internationalization of franchises; after school services.

\section{Introducción}

Las franquicias son un modelo de negocio que participa activamente en la economía de muchos países; en Estados Unidos, por ejemplo, operan cerca de 773.603 establecimientos generando más de ocho millones de empleos con una participación del $4.7 \%$ del PIB (International Franchise Association, 2019), por su parte, los países más desarrollados de Europa reúnen cerca de 390.000 establecimientos, representando más de 6200 marcas entre locales y extranjeras (European Franchise Federation, 2018).

El crecimiento sostenido del mercado de franquicias en países desarrollados, posicionando marcas fuertes alrededor del mundo, impulsa el afianzamiento de este formato empresarial en mercados emergentes. En Latinoamérica, la creación de franquicias es un proceso en auge, en principio porque es un territorio no saturado (Lanchimba \& Medina, 2018) lo que despierta el interés de franquicias extranjeras deseosas de posicionar sus marcas en nuevos mercados, $y$, de otra parte, por la positiva contribución de las franquicias al desarrollo de los países ya que al exportar modos de operación, coordinación y dirección para los negocios se impulsa al desarrollo y la creación de oportunidades en aquellos sectores donde se desenvuelve la franquicia (Michael, 2014)

En lo que se refiere a la exportación del negocio, la literatura académica que ha estudiado los 
Agudelo Cotes, Karen J.; Bedoya Gómez, Diana C.

Riesgos de adaptación en franquicias: herramientas de contabilidad de gestión para mitigarlos

procesos de internacionalización de franquicias generalmente se enfoca en la perspectiva del franquiciador (Rosado et al. 2018) señalando la importancia para este de desarrollar capacidades que permitan monitorear a los franquiciados extranjeros y controlar comportamientos oportunistas y así aumentar la probabilidad de éxito de las franquicias (Shane, 1996) o, como poseer actitudes positivas, tolerancia al riesgo, orientación internacional y mentalidad abierta son cualidades relevantes que el franquiciador debe autoevaluar en su proceso a la internacionalización (Eroglu, 1992).

En cuanto al riesgo, decidir internacionalizar una marca requiere, una valoración seria por parte del franquiciador de los costos vs beneficios percibidos, y, desarrollar plataformas de gestión para la planeación y la puesta en marcha de incursionar en el extranjero (Eroglu, 1992).

En general un grueso componente de la literatura académica sobre los procesos de internacionalización se enfocan en las características, actitudes y procesos a desarrollar por parte del franquiciador, sin embargo, los procesos de franquicia involucran al franquiciador $y$ al franquiciado por lo que generar un ambiente cooperativo que permita trabajar en el éxito del proceso, acarreará beneficios y satisfacciones mutuas, por tanto, un moderno enfoque para analizar los procesos de internacionalización requiere hacerse desde las dos partes implicados (Rosado et al. 2018).

En Colombia la información sobre el desarrollo de franquicias es escasa. La (Cámara Colombiana de Franquicias, 2018), estima que, en el país se han establecido a 2018 más de 500 marcas que operan con un número aproximado de 12.000 negocios. Sin embargo, no existe un marco normativo específico para este modelo empresarial, además de poca documentación estadística o académica que describa con certeza la caracterización de esta dinámica de negocio en el país, tanto para marcas locales, como para marcas extranjeras, sus procesos de expansión y adaptación.

En este sentido, el estudio propone el análisis de variables que pueden afectar el éxito de la franquicia en Colombia, estableciendo los riesgos de adaptación potenciales para la marca y de esta manera contribuir al acervo académico y a la comprensión del fenómeno del desarrollo de franquicias en el país. Aun cuando el estudio se centra en empresas que prestan servicios de formación extracurricular para niños; los hallazgos del trabajo, podrían utilizarse y validarse en diferentes conceptos de franquicia. Otro elemento que incorpora este documento, es la propuesta de un modelo conceptual de aplicación de herramientas de contabilidad de gestión que ayude a mitigar los riesgos de adaptación identificados.

Los riesgos de adaptación asociados a la franquicia, bien pueden hacer uso de las estrategias y herramientas que utiliza la contabilidad de gestión tanto para la administración de los resultados y los recursos de la red de empresas franquiciadas, así como para establecer una fuente de información confiable para la toma de decisiones estratégicas y tácticas que maximicen los beneficios de las empresas involucradas, debido a que la Contabilidad de Gestión, es entendida como aquella rama de la contabilidad encargada de medir y analizar la información para controlar y racionalizar los recursos, que facilita la planeación, los procesos de toma de decisiones y el logro de los propósitos económicos esperados, involucrando 
datos financieros y no financieros (Osorio et al. 2015); evoluciona y se adapta conforme se transforman las dinámicas empresariales en consonancia con circunstancias propias del negocio y de los contextos económicos, sociales y culturales (Salgado, 2014), el estudio sostiene que una adecuada implementación de herramientas que integran los procesos de la contabilidad de gestión minimizarían los riesgos identificados en los modelos de franquicia base de estudio.

Sobre la implementación de herramientas y de los conceptos de contabilidad de gestión en los modelos de franquicia y sus posibles resultados, no fueron encontrados para el caso colombiano.

En el escenario internacional, se encontró que en España la (Asociación Española de Contabilidad y Administración de Empresas -AECA, 2008) a través del documento Nro. 35 de 2008 denominado La Contabilidad de Gestión en las Empresas Franquiciadoras, presenta una caracterización de las empresas de franquicia en ese país, y se establece la incorporación de elementos de contabilidad de gestión, que aportan a su adecuado funcionamiento. Ese documento señala que un adecuado ejercicio de planeación estratégica puede minimizar las divergencias que se presentan entre franquiciador $y$ franquiciado. Dentro de las etapas de planeación estratégica, señaladas en el documento, la contabilidad de gestión está presente en la fase relacionada con el diseño de un sistema de información soportado en los presupuestos y en la contabilidad de costos, y posteriormente en la fase reservada al diseño de un sistema de evaluación, seguimiento y control se establece la implementación de un cuadro de mando integral y de indicadores de gestión.

Otro documento relacionado con la gestión en la industria de la franquicia lo desarrolla (Cordobés \& Soldevila, 2015), las autoras, expresan la necesidad de las organizaciones de establecer nuevas y mejoradas herramientas de control de la gestión, que les permita sobrevivir en los actuales entornos competitivos y cambiantes.

Por su parte (Ramírez et al. 2013) comprendiendo el impacto que genera sobre una red de franquicias, los comportamientos oportunistas de cualquiera de sus miembros, estudiaron en 240 empresas franquiciadoras de España, como el diseño y uso de sistemas de contabilidad de gestión, ayudan al control de las franquicias, encontrando además que apoyándose en la información derivada de los sistemas de contabilidad de gestión se favorecían los procesos de aprendizaje $y$ el relacionamiento entre franquiciados y franquiciadores.

Otros factores también son considerados como elementos cruciales para el éxito de los modelos de franquicia, además del uso de información de tipo cuantitativo derivados de los informes financieros, se trata de los temas relacionados con el servicio al cliente, administración del personal (Luangsuvimol \& Kleiner, 2004) y la construcción de relaciones de confianza (Wu, 2015) todas ellas reconocidas como innovaciones de la contabilidad de gestión.

A partir de lo expuesto, el presente artículo es un estudio exploratorio, que identifica los riesgos asociados a la adaptación de franquicias internacionales dedicadas a la prestación servicios de formación extracurricular para niños en Colombia. El ejercicio 
Agudelo Cotes, Karen J.; Bedoya Gómez, Diana C.

Riesgos de adaptación en franquicias: herramientas de contabilidad de gestión para mitigarlos

exploratorio establece, en una primera parte, la definición de variables que caracterizan el modelo de negocio de la marca y la etapa de desarrollo en la que se encuentra, y una segunda, el planteamiento de un modelo conceptual de aplicación de contabilidad de gestión que ayude a mitigar los riesgos identificados y permitir una adaptación exitosa. El proceso se llevó a cabo a partir de archivos, fuentes secundarias y entrevistas a profundidad realizadas a los líderes responsables del proceso de expansión de la franquicia en Colombia.

El presente documento está organizado en cuatro secciones, incluida esta introducción, una segunda sección que contiene la caracterización de cada marca a partir de la descripción de las variables estudiadas y la identificación de los riesgos asociados a estos modelos de franquicia, la tercera sección expone la propuesta conceptual para la aplicación de herramientas de contabilidad de gestión, y, por último, las conclusiones del artículo.

\section{Adaptación de las franqui- cias de servicios}

El desarrollo de la investigación se centra en los riesgos de adaptación de empresas de franquicia que prestan servicios de formación extracurricular para niños. En Colombia, es cada vez mayor la oferta de servicios de formación extracurricular para niños como complemento a la formación académica que reciben en los colegios. Una buena parte de la oferta la canalizan los colegios que ponen a disposición de los padres un amplio portafolio de actividades culturales, artísticas y deportivas que los niños pueden hacer después de terminada la jornada escolar a razón de una tarifa mensual adicional a la colegiatura. Esta es una alternativa muy interesante para los padres debido a que la actividad se realiza en las instalaciones del centro educativo evitando desplazamientos y bajo el esquema de seguridad y ambiente controlado que los padres conocen.

Otra modalidad común de clase extracurricular son los refuerzos académicos a cargo de profesores de matemáticas, inglés $u$ otras asignaturas, con clases privadas en los hogares para mejorar el rendimiento de los niños. Y, por último, pero no menos significativo, en cuanto a crecimiento y amplitud de la oferta, son los negocios de franquicia especializados en ciertas áreas de conocimiento que actúan como un complemento educativo para fortalecer aptitudes académicas de los estudiantes.

La mayoría de estas franquicias, establecen métodos ingeniosos, creativos, diseñados pedagógicamente, buscando una propuesta de valor más atractiva para padres e hijos; la presencia de estos establecimientos en Colombia se ha detectado a partir de la información del directorio especializados de franquicias de la (Feria Internacional de Negocios y Franquicias, 2019), que en el país hay alrededor de 5 marcas, todas extranjeras que han desplegado su modelo de franquicia especialmente en las principales ciudades del país, como son Bogotá DC, Medellín, Barranquilla y Cali.

Teniendo en cuenta que los procesos de internacionalización de una franquicia dependen de ciertos factores relacionados con variables macro y micro económicas, factores culturales y de contexto político y legal (Rosado et al. 2018) y; así mismo la práctica de la contabilidad de gestión no posee un 
marco unificado o definido de acción y su implementación obedece a las necesidades de la organización, el ciclo de desarrollo en el que se encuentre y del nivel de control que se requiere (Oriol et al. 2014), fue indispensable para el desarrollo del estudio, un reconocimiento previo de las características de operación de las empresas y conforme a la configuración de su modelo de gestión, identificar los riesgos que supone la adaptación de la marca y así proponer un modelo conceptual de contabilidad de gestión que satisfaga su propósito de afianzamiento.

Para esto, un primer acercamiento a la caracterización de las franquicias se obtuvo de fuentes secundarias ubicadas en páginas web de las franquicias, noticias, programas de actualidad, revistas y videos en la internet. Como es habitual en el modelo de franquicia, el establecimiento de acuerdos de confidencialidad, no fue posible acceder a documentos privados, tales como contratos, manuales de operación, procedimientos, presupuestos o acceso a la tecnología utilizada y manteniendo esta condición de reserva, a continuación, se enuncian en el Cuadro 1 las características generales de las marcas, sin mencionar sus nombres reales.

\section{Cuadro 1}

\section{Características generales de las marcas}

\begin{tabular}{|c|c|c|c|}
\hline $\begin{array}{c}\text { CARACTERISTICAS / } \\
\text { MARCAS }\end{array}$ & 1 & 2 & 3 \\
\hline Origen de la marca & Extranjera & Extranjera & Extranjera \\
\hline $\begin{array}{l}\text { Nro. Establecimientos en } \\
\text { Colombia }\end{array}$ & 6 & 0 & 5 \\
\hline $\begin{array}{l}\text { Apertura mercado } \\
\text { colombiano }\end{array}$ & 2015 & 2012 & 2015 \\
\hline Concentración & Idiomas / matemáticas & Idiomas & Ciencias \\
\hline Modelos de operación & $\begin{array}{l}\text { 1. Academias de } \\
\text { formación que operen } \\
\text { dentro de colegios. } \\
\text { 2. Franquicia personal }\end{array}$ & $\begin{array}{l}\text { 1. Centros de } \\
\text { formación. } \\
\text { 2. Franquicia } \\
\text { personal. }\end{array}$ & $\begin{array}{l}\text { 1. Franquicia móvil } \\
\text { 2. Centros de formación } \\
\text { 3. Eventos masivos - } \\
\text { temporada vacacional - } \\
\text { cumpleaños y campamentos. }\end{array}$ \\
\hline $\begin{array}{l}\text { Mecanismo de entrada de } \\
\text { la marca }\end{array}$ & Franquiciado Máster & Franquiciado máster & Franquiciado máster \\
\hline $\begin{array}{l}\text { Estrato socioeconómico al } \\
\text { que se dirige }\end{array}$ & 4. 5 y 6 & 5 y 6 & 4,5 y 6 \\
\hline
\end{tabular}

Fuente: Elaboración propia (2020)

Se observa que las marcas referenciadas ofertan sus servicios para los estratos socioeconómicos más altos de la población y sus mecanismos de entrada se soportan sobre la estrategia de franquiciado máster, lo que significa el otorgamiento de derechos de explotación del formato de negocio en un territorio amplio, en los casos observados implica el desarrollo de la marca en todo el 
Agudelo Cotes, Karen J.; Bedoya Gómez, Diana C.

Riesgos de adaptación en franquicias: herramientas de contabilidad de gestión para

mitigarlos

territorio nacional.

Los elementos de caracterización se establecieron con base en dos momentos, el primer momento de trabajo colectivo entre franquiciado y franquiciador está relacionado con las actividades de pre-apertura de las unidades, el segundo momento, son las actividades propias de la franquicia en funcionamiento, en el siguiente Cuadro 2 se esquematiza las variables que orientaron la exploración del proceso de adaptación en la franquicia:

\section{Cuadro 2}

\section{Variables para caracterizar el modelo de negocio de la franquicia}

\begin{tabular}{|c|c|}
\hline ETAPAS & VARIABLES \\
\hline \multirow{4}{*}{ Previa a la apertura } & Promoción Marca \\
\hline & Selección de candidatos \\
\hline & Adecuación / infraestructura \\
\hline & Entrenamiento \\
\hline \multirow{7}{*}{ Después de la apertura } & Soporte \\
\hline & Auditorías \\
\hline & Internacionalización \\
\hline & Ingresos \\
\hline & Estructura de costos \\
\hline & Precios \\
\hline & Resolución de conflictos \\
\hline
\end{tabular}

Fuente: Elaboración propia (2020)

A continuación, el Cuadro 3 franquicias estudiadas a partir de las describe las características de las

variables definidas. 


\section{Cuadro 3 \\ Caracterización de las marcas}

\begin{tabular}{|c|c|c|c|}
\hline $\begin{array}{l}\text { VARIABLES / } \\
\text { MARCAS }\end{array}$ & 1 & 2 & 3 \\
\hline $\begin{array}{c}\text { Promoción de la } \\
\text { marca }\end{array}$ & $\begin{array}{l}\text { La estrategia comunicacional y } \\
\text { promocional para dar a conocer } \\
\text { la marca y acceder a futuros clien- } \\
\text { tes, está centrada en el manejo } \\
\text { de redes sociales y páginas web } \\
\text { especializadas en franquicia. Las } \\
\text { páginas y canales promocionales } \\
\text { son seleccionadas por el franqui- } \\
\text { ciado máster en cada país. Otra } \\
\text { estrategia es la participación en } \\
\text { ferias y eventos para un público } \\
\text { segmentado como padres de fami- } \\
\text { lias, colegios y niños, sin embargo, } \\
\text { durante los años (5) que lleva la } \\
\text { franquicia en Colombia, los resul- } \\
\text { tados obtenidos no compensan la } \\
\text { inversión realizada }\end{array}$ & $\begin{array}{l}\text { Para la promoción de } \\
\text { la marca el franqui- } \\
\text { ciado recibe todo un } \\
\text { entrenamiento orien- } \\
\text { tado a un proceso de } \\
\text { comunicación efectiva } \\
\text { con sus potenciales } \\
\text { clientes, así como es- } \\
\text { trategias para captar } \\
\text { clientes. La casa ma- } \\
\text { triz gestiona campañas } \\
\text { promocionales a tra- } \\
\text { vés de redes sociales y } \\
\text { publicita sus diferentes } \\
\text { establecimientos en el } \\
\text { mundo. }\end{array}$ & $\begin{array}{l}\text { Desde la casa matriz } \\
\text { no se definieron estra- } \\
\text { tegias en este aspecto, } \\
\text { el franquiciado utilizó } \\
\text { la promoción vía redes } \\
\text { sociales y visitas direc- } \\
\text { tas a los colegios. }\end{array}$ \\
\hline $\begin{array}{l}\text { Selección de can- } \\
\text { didatos }\end{array}$ & $\begin{array}{l}\text { Desde casa matriz se ha definido } \\
\text { un perfil básico, cuyas caracterís- } \\
\text { ticas esenciales es que el futuro } \\
\text { franquiciado se identifique como } \\
\text { un emprendedor, con interés y } \\
\text { capital para dedicarse a su propio } \\
\text { negocio }\end{array}$ & $\begin{array}{l}\text { Se define como per- } \\
\text { sonas o empresas por } \\
\text { gusto por la educación } \\
\text { especialmente por la } \\
\text { educación para niños. } \\
\text { Es importante que la } \\
\text { persona o represen- } \\
\text { tante de la empresa } \\
\text { se desenvuelva con un } \\
\text { alto nivel de inglés, de- } \\
\text { bido a que gran parte } \\
\text { de las interacciones y } \\
\text { comunicaciones con la } \\
\text { casa matriz se harán } \\
\text { en esa lengua. }\end{array}$ & $\begin{array}{l}\text { Se perfilan personas } \\
\text { motivadas por la idea } \\
\text { de emprender su propio } \\
\text { negocio. }\end{array}$ \\
\hline $\begin{array}{l}\text { Adecuación de } \\
\text { infraestructura }\end{array}$ & $\begin{array}{l}\text { En el modelo de academia los } \\
\text { franquiciados son acompañados } \\
\text { por el franquiciado máster para } \\
\text { la selección y ubicación del local } \\
\text { y orientados sobre el acondiciona- } \\
\text { miento con el que este debe cum- } \\
\text { plir. En cuanto a los otros modelos } \\
\text { de operación de la franquicia, no } \\
\text { existen requisitos específicos so- } \\
\text { bre instalaciones o adecuaciones }\end{array}$ & $\begin{array}{l}\text { La franquicia entrega } \\
\text { un manual de imagen } \\
\text { en el que se estable- } \\
\text { cen las condiciones de } \\
\text { infraestructura, mobi- } \\
\text { liario, adecuaciones y } \\
\text { decoración que deben } \\
\text { evidenciarse en cada } \\
\text { centro alrededor del } \\
\text { mundo. Adicionalmen- } \\
\text { te a través de videos } \\
\text { instructivos los fran- } \\
\text { quiciados conocen el } \\
\text { concepto visual que } \\
\text { impera en cada centro. }\end{array}$ & $\begin{array}{l}\text { No era necesario, por } \\
\text { tratarse de franquicias } \\
\text { personales que se des- } \\
\text { plazaban con el equipo } \\
\text { requerido }\end{array}$ \\
\hline
\end{tabular}


Agudelo Cotes, Karen J.; Bedoya Gómez, Diana C.

Riesgos de adaptación en franquicias: herramientas de contabilidad de gestión para mitigarlos

\begin{tabular}{llll}
\hline $\begin{array}{l}\text { VARIABLES / } \\
\text { MARCAS }\end{array}$ & 1 & 2 & 3 \\
\hline
\end{tabular}

La franquicia progra-

ma entrenamientos

a lo largo del año, alrededor de cuatro (4) eventos de capacita-

A través de videos interactivos se realiza la capacitación sobre el manejo de la plataforma On-line, que involucra procesos de inscrip-

Entrenamiento ción, asignación de cuentas a los alumnos, facturación, seguimiento de clientes para evaluar las estrategias de marketing. Todo el proceso de entrenamiento es virtual.

La relación de acompañamiento entre la casa matriz y el franquiciado máster es permanente, en gran

Soporte medida por la relación personal que se construye entre las partes y por la proactividad del franquiciado máster ción anual. El entrenamiento que se recibe se orienta sobre las áreas de mercadeo y administración de la franquicia, introduciendo al franquiciado en el manejo del software de gestión con el que cuenta la franquicia para soportar y acompañar los procesos de admisión, inventarios de materiales e ingresos.

Además del software de gestión que posee

la casa matriz y con la que interactúan los franquiciados, están los manuales de operación y una serie de videos y orientación virtual que acompañan al franquiciado al desarrollo de la franquicia, se tiene un ejecutivo de cuenta ubicado en la sede principal quien mantiene una comunicación semanal con los franquiciados masters para atender sus necesidades, realizar seguimiento y acompañar al franquiciado.

Una vez al año, se establece un proceso de evaluación conjunta entre el franquiciado y un vicepresidente de la marca; este proceso implica que el franquiciado evalúa el soporte y acompañamiento recibido durante el año por parte de la casa matriz a través del ejecutivo de cuenta asignado
Se realizaron entrenamientos orientados a la transferencia del componente pedagógico que se configura tras el trabajo lúdico de la metodología de formación. Importante el dominio del idioma inglés para las comunicaciones con la casa matriz y para comprender las instrucciones definidas en cada una de las etapas de construcción del modelo.
Todos los modelos de formación se pueden descargar de la plataforma, con instrucciones claras para el manejo de cada estrategia lúdica.
No se realizaron visitas de seguimiento, básicamente el control de ventas, depende del reporte que realizan los franquiciados tán contemplados a manera de política en la franquicia. 


\begin{tabular}{|c|c|c|c|}
\hline $\begin{array}{l}\text { VARIABLES / } \\
\text { MARCAS }\end{array}$ & 1 & 2 & 3 \\
\hline Internacionalización & $\begin{array}{l}\text { La franquicia en su proceso de } \\
\text { internacionalización no tiene defi- } \\
\text { nido elementos de evaluación, de } \\
\text { comprensión o caracterización del } \\
\text { mercado al que se incorpora }\end{array}$ & $\begin{array}{l}\text { La casa matriz, ha } \\
\text { realizado estudios } \\
\text { identificando potencia- } \\
\text { les zonas para la ex- } \\
\text { pansión de la marca, } \\
\text { basándose en el cre- } \\
\text { cimiento económico de } \\
\text { la región y la demanda } \\
\text { que se tenga del servi- } \\
\text { cio que se ofrece. Co- } \\
\text { lombia da señales de } \\
\text { interés en nuestra área } \\
\text { evidenciado a través } \\
\text { de la creciente oferta } \\
\text { de cursos. }\end{array}$ & \\
\hline
\end{tabular}

Para la franquicia los ingresos se generan Los ingresos periódicos lo consti- vía regalías derivatuyen el pago de una tarifa esta- do de las matrículas blecida que se liquida por cada y mensualidades de usuario registrado en la plataforma cada estudiante inscrito en el establecimiento

Las unidades o el franquiciado máster no son orientados o no se tienen disposiciones sobre estructuras mínimas o máximas de costo.
El franquiciado es autónomo para construir la estructura de costos que mejor proyecte las necesidades y operación del negocio corresponde a un \% de los valores recaudados por concepto de matrículas y niños inscritos en las actividades de formación

Cada unidad conforme a las características de la zona que atiende, establece su propia estructura de costos, en la que básicamente se debe tener en cuenta costos de transporte y promoción.

\begin{tabular}{|c|c|c|c|}
\hline Precio & $\begin{array}{l}\text { Los precios para las franquicias } \\
\text { máster en el mundo son definidos } \\
\text { por casa matriz y de cierta forma } \\
\text { son estandarizados, en Colombia } \\
\text { particularmente esta franquicia ha } \\
\text { detectado fluctuaciones significati- } \\
\text { vas en la percepción y accesibili- } \\
\text { dad de las familias entre una ciu- } \\
\text { dad y otra. En términos generales } \\
\text { no es un proceso estructurado. }\end{array}$ & $\begin{array}{l}\text { Los franquiciados en } \\
\text { cada lugar donde se } \\
\text { desarrolle la franqui- } \\
\text { cia y con base en las } \\
\text { condiciones del mer- } \\
\text { cado son autónomos } \\
\text { en la fijación de pre- } \\
\text { cios de las matrículas } \\
\text { y mensualidades. Un } \\
\text { promedio para la fran- } \\
\text { quicia conforme a los } \\
\text { estudios realizados por } \\
\text { la casa matriz, estable- } \\
\text { cen el precio entre los } \\
\text { US } \$ 60 \text { y US } \$ 120\end{array}$ & $\begin{array}{l}\text { Se establece conforme } \\
\text { a la ciudad donde se } \\
\text { desarrolla la franquicia } \\
\text { y atendiendo los valo- } \\
\text { res promedio que se } \\
\text { encuentran en el mer- } \\
\text { cado para la oferta de } \\
\text { productos similares. }\end{array}$ \\
\hline
\end{tabular}


Agudelo Cotes, Karen J.; Bedoya Gómez, Diana C.

Riesgos de adaptación en franquicias: herramientas de contabilidad de gestión para mitigarlos

\begin{tabular}{|c|c|c|c|}
\hline $\begin{array}{l}\text { VARIABLES / } \\
\text { MARCAS }\end{array}$ & 1 & 2 & 3 \\
\hline $\begin{array}{l}\text { Resolución de } \\
\text { conflictos }\end{array}$ & $\begin{array}{l}\text { Con los clientes no existen proto- } \\
\text { colos. Y no hay seguimiento al gra- } \\
\text { do de satisfacción de los clientes. } \\
\text { Depende mucho de la proactividad } \\
\text { de los franquiciados y de sus ha- } \\
\text { bilidades para resolver situaciones } \\
\text { complejas }\end{array}$ & $\begin{array}{l}\text { Los clientes deben } \\
\text { escalar sus inconfor- } \\
\text { midades a través del } \\
\text { conducto regular, esto } \\
\text { es directamente con } \\
\text { el franquiciado y pos- } \\
\text { teriormente si sus du- } \\
\text { das no son resueltas a } \\
\text { través del franquiciado } \\
\text { máster de la región }\end{array}$ & $\begin{array}{l}\text { En los contratos se es- } \\
\text { tablecen procedimien- } \\
\text { tos para el tratamiento } \\
\text { de situaciones difíciles } \\
\text { que, durante la exis- } \\
\text { tencia de la franquicia } \\
\text { en Colombia, no se han } \\
\text { llegado a presentar. }\end{array}$ \\
\hline
\end{tabular}

Fuente: Elaboración propia (2020)

\section{Riesgos en los procesos de adaptación de franquicias}

Con base en la caracterización de los modelos de negocios, se presenta a continuación Cuadro 4 Riesgos Identificados para las marcas que ponen en peligro la adaptación de las mismas.

\section{Riesgos Identificados para las marcas}

\begin{tabular}{|c|c|c|c|}
\hline & ACTIVIDADES & & RIESGOS \\
\hline \multirow{4}{*}{$\begin{array}{l}\text { Procesos previos a } \\
\text { la apertura }\end{array}$} & Promoción Marca & 1. & Estrategias estériles \\
\hline & Selección de candidatos & 2. & Perfiles inadecuados \\
\hline & Entrenamiento & 3. & Áreas sin entrenamiento \\
\hline & Adecuación / Infraestructura & 4. & No se consideran riesgos. \\
\hline \multirow{6}{*}{$\begin{array}{l}\text { Procesos posterio- } \\
\text { res a la apertura }\end{array}$} & Soporte & 5. & $\begin{array}{l}\text { Tecnología con deficiencias / Comunica- } \\
\text { ción débil }\end{array}$ \\
\hline & Auditorías & 6. & Falta de seguimiento \\
\hline & Internacionalización & 7. & $\begin{array}{l}\text { Inadecuada identificación de oportunida- } \\
\text { des }\end{array}$ \\
\hline & Ingresos / precios & 8. & $\begin{array}{l}\text { Precios no competitivos / Valoración de } \\
\text { regalías }\end{array}$ \\
\hline & Estructura de costos & 9. & $\begin{array}{l}\text { Estructura inadecuada de costos y gastos } \\
\text { para las unidades }\end{array}$ \\
\hline & Resolución de conflictos & 10. & $\begin{array}{l}\text { Pérdida de clientes / Afectación a la } \\
\text { imagen de la marca }\end{array}$ \\
\hline
\end{tabular}

Fuente: Elaboración propia (2020) 
En el Cuadro 5 se enuncian para cada uno de los riesgos en la adaptación de franquicias, los hallazgos relacionados para las marcas objeto de estudio.

\section{Cuadro 5 \\ Descripción de riesgos}

\begin{tabular}{|c|c|c|}
\hline RIESGOS & DESCRIPCIÓN & HALLAZGOS DEL ESTUDIO \\
\hline Estrategias estériles & $\begin{array}{l}\text { Se convierten en inversiones que no } \\
\text { concretan la apertura de nuevas unidades, } \\
\text { lo cual conlleva a la falta de clientes y el } \\
\text { quiebre de la franquicia. }\end{array}$ & $\begin{array}{l}\text { Este es un aspecto sensible, las franquicias de } \\
\text { servicios de formación extracurricular para niños, } \\
\text { están compitiendo actualmente en un mercado } \\
\text { con mucha oferta, y los nombres de estas marcas } \\
\text { extranjeras son desconocidos. El trabajo conjunto } \\
\text { entre casa matriz y franquiciado en este aspecto } \\
\text { es esencial y no se evidencia en las franquicias } \\
\text { estudiadas. }\end{array}$ \\
\hline
\end{tabular}

Del proceso de selección de candidatos, depende la sostenibilidad de las unidades franquiciadas y por ende el crecimiento y fortalecimiento de la red. Aun cuando el franquiciado está invirtiendo su capital y no tenga la intención de destruir su inversión, es probable que desconozca o carezca de las habilidades necesarias para lograr la adaptación de la franquicia.

En las franquicias analizadas la selección de perfil es muy básica, esencialmente se requiere que el futuro franquiciado cuente con capital e interés en desarrollar su propio negocio. Pero, sin la adecuada capacidad para gestionar el mercado, buscar clientes y promocionar la marca; el deseo y el recurso económico serán insuficientes.

\begin{tabular}{ll}
\hline & Cuando los franquiciados no completan \\
un proceso de capacitación exitoso, se \\
generarán dificultades en la aplicación \\
del modelo desde su puesta en marcha \\
inicial. Siendo vital para las franquicias \\
Áreas sin entrenamiento $\quad$ el manejo de plataformas tecnológicas, \\
debe garantizarse que se entiende y \\
se comprende todas y cada una de las \\
posibilidades y las características de la \\
herramienta tecnológica y del concepto de \\
negocio.
\end{tabular}

Para desplegar el modelo de negocio, no se requiere esencialmente la consecución y adecuación de inmuebles, forzar su implementación por parte de la casa matriz, puede implicar en inversiones innecesarias y congelando recursos importantes para
Todas las franquicias cuentan con procesos de entrenamiento y por tratarse de modelos que operan ya con éxito en muchos países, es muy seguro y confiable la transmisión de las características metodológicas y técnicas. No obstante, el entrenamiento operacional del modelo de negocio podría no ser suficiente, cuando otras áreas, como por ejemplo la administración de personal, manejo de situaciones de conflicto, búsqueda de mercados y estrategias de comercialización, no se incluyen amplia y permanentemente en el entrenamiento. atender aspectos más cruciales.

Infraestructura innecesaria
Las marcas estudiadas han encontrado en la franquicia personal una mejor forma de conectar con su perfil de clientes, por tanto, las infraestructuras han resultado innecesarias y la casa matriz ha flexibilizado los aspectos relacionados con la creación de centros para favorecer la adaptación de las franquicias. En este sentido no se identifica como un riesgo que desencadene en el fracaso del modelo.

* Franquicias personales, derechos entregados a una persona natural para que opere directamente la marca.

Hacer un seguimiento permanente a la plataforma, implica revisar los soportes lógicos y copias de respaldo sobre los Tecnología con deficiencias datos, garantizar agilidad de la conectividad / Comunicación débil e interfaz limpia e intuitiva para todos los perfiles de usuarios. La falta de comunicación es una de las mayores complejidades del modelo de franquicia.
Algunas franquicias mantienen contacto directo y permanente con agentes de cuenta o la casa matriz, sin embargo, el elemento esencial para este relacionamiento es el manejo de una segunda lengua, especialmente el inglés. Aspecto que debe sumarse en el perfil del franquiciado. 


\section{Cont... Cuadro 5}

\begin{tabular}{|c|c|c|}
\hline RIESGOS & DESCRIPCIÓN & HALLAZGOS DEL ESTUDIO \\
\hline Falta de seguimiento & $\begin{array}{l}\text { Al no realizarse procesos de verificación } \\
\text { se pueden generar malos manejos de la } \\
\text { marca, una apropiación inadecuada sobre } \\
\text { la forma de prestación del servicio, la } \\
\text { transferencia de los métodos de formación } \\
\text { al usuario final, y desconocimiento del } \\
\text { estado de desarrollo de las unidades. }\end{array}$ & $\begin{array}{l}\text { Las franquicias revisadas han recibido escaso } \\
\text { seguimiento en el funcionamiento de su } \\
\text { operación, adicionalmente en la mayoría de los } \\
\text { casos la generación de ingresos se deriva de los } \\
\text { usuarios registrados en la plataforma, por tanto, } \\
\text { no es redundante diseñar más de una estrategia } \\
\text { de seguimiento para garantizar la transparencia } \\
\text { de los registros. }\end{array}$ \\
\hline
\end{tabular}

\begin{tabular}{|c|c|c|}
\hline $\begin{array}{l}\text { Inadecuada identificación } \\
\text { de oportunidades }\end{array}$ & $\begin{array}{l}\text { Se debe garantizar desde casa matriz un } \\
\text { estudio completo de las futuras zonas } \\
\text { de expansión, para garantizar una fácil } \\
\text { adaptación del modelo y evitar pérdida } \\
\text { de valor de la marca y el posterior } \\
\text { incumplimiento en las metas de cobertura } \\
\text { y crecimiento. }\end{array}$ & $\begin{array}{l}\text { Aspectos como la regionalización, la cultura y los } \\
\text { micro-ecosistemas económicos de las ciudades } \\
\text { se convierten en la principal barrera para la } \\
\text { adaptación y crecimiento de la franquicia y sobre } \\
\text { el particular no se destaca la realización de un } \\
\text { análisis detallado y minucioso de la casa matriz o } \\
\text { de los franquiciados sobre su zona de influencia. }\end{array}$ \\
\hline
\end{tabular}

La variable precio es determinante para garantizar un flujo de ingresos constantes, dadas las características de los mercados en los que se inserta la franquicia, políticas no adecuadas de precio puede disminuir la competitividad de la marca, puede arriesgar la percepción de valor y no generar los ingresos necesarios para la rentabilidad y la operación del negocio.
Los precios de referencia de estas franquicias en sus países de origen tienen un nivel más alto de lo que este mercado puedo soportar en Colombia, si bien cada unidad con base en el estudio de su público focal puede determinar los precios, la relación costo-beneficio se pone en riesgo debido a los costos promocionales, de posicionamiento, pago por materiales $y$ transporte, que otras ofertas no contemplan y que resultaran más atractivas para los padres de familia por su asequibilidad.
Estructura inadecuada de costos y gastos para las unidades
Cada unidad y franquiciado máster es autónomo en el manejo de sus operaciones administrativas, no obstante, para consolidar resultados satisfactorios que facilite el progreso de la unidad y de estas en la red, conviene el establecimiento de indicadores de gestión mínimos y estandarizados para cada unidad.
Las unidades han establecido su estructura de costos y de indicadores, respondiendo a las necesidades de información financiera establecida normativamente, en el caso de aquellas constituidas como empresa. Las que operan como persona natural, no poseen un sistema de información que les permita identificar con certeza el margen o rentabilidad de su negocio.
Es una variable crítica para las unidades, y para la generación de ingresos de la marca, un cliente enojado insatisfecho Pérdida de clientes / en la entrega de valor que propone la Afectación a la imagen de marca, puede convertirse en el voz a voz la marca de una mala imagen, en consecuencia es indispensable estandarizar el manejo de conflictos con los clientes y con los franquiciados, de lo contrario todo esfuerzo para posicionar la marca será infructuoso.
Durante el desarrollo de este artículo, ninguna de las franquicias estudiadas había presentado problemas relacionados con inconformidades con clientes.

Fuente: Elaboración propia (2020) 
Los riesgos identificados anteriormente se agruparon en dos conceptos: 1) Riesgo de Desarrollo comercial; este riesgo circunscribe otros riesgos anteriormente descritos como perfiles inadecuados, estrategias estériles para la promoción de la marca, precios no competitivos, pérdida de clientes, afectación de la marca. Para la facilitar la adaptación de la marca el proceso de internacionalización requiere estudios profundos sobre las particularidades culturales $y$ microeconómicas de la zona geográfica en la que se desea insertar el negocio, en Colombia las dinámicas económicas y de comportamiento son muy distintas entre ciudades, por tanto, precios y costos deben fluctuar y adaptarse para hacer viables las unidades franquiciadas.

El estudio de las particularidades culturales y micro-económicas, arrojará ideas claras sobre el perfil de franquiciado que se requiere para hacer frente a los retos, un emprendedor proactivo, que conoce e interactúa cómodamente con el mercado, especialmente el mercado objetivo, es una ventaja deseable para el éxito de la adaptación. Lo anterior concuerda con lo planteado por (Shanne \& Spell, 1998) cuando sugieren que la probabilidad de éxito de nuevas franquicias mejora cuando se buscan franquiciados a quienes delegar funciones relacionadas con la búsqueda de nuevos puntos, y, de otra parte, el franquiciado sopesara la decisión de invertir en una u otra franquicia en la medida que esta sea capaz de desarrollar marcas fuertes.

2) Riesgo de Animación de la red, contiene los riesgos asociados a la falta de seguimiento, $y$, la detección de problemas de comunicación y de soporte entre las unidades y la casa matriz.

El acercamiento permanente a la unidad entendido como una estrategia de relacionamiento, es una ventaja en dos sentidos, de una parte, se gestan canales de comunicación ininterrumpidos, lo que genera una sensación de soporte y seguridad en el franquiciado y de otra, es ideal para ejercer cierto control sobre el desempeño, acorde a (Lanchimba \& Medina, 2018) que sugiere que administrar el control sobre las franquicias se sustenta en relaciones de confianza, satisfacción y comunicación mutua.

El diagrama 1 Agrupación de Riesgos, señala la integración de riesgos y con base en estas dos categorías, se plantearan herramientas de contabilidad de gestión para mitigarlos. 


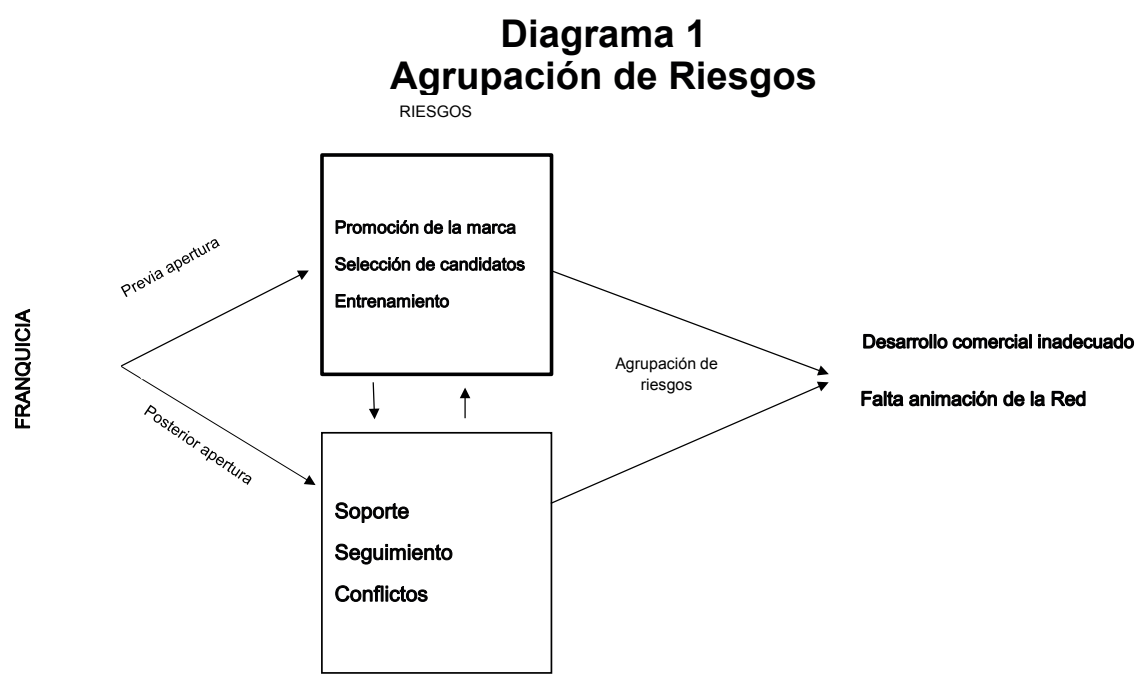

Fuente: Elaboración propia (2020)

\section{Herramientas de contabilidad de gestión para las franquicias}

Los procesos de adaptación de franquicia, son un fenómeno poco estudiado por la literatura académica en Colombia, aun cuando este desarrollo empresarial está tomando fuerza y se populariza entre las estrategias de creación de empresa. Cuando un franquiciado motivado por el éxito de la marca en otros países y con base en la evolución del mercado local, decide invertir en una franquicia, opta por aquellas que mejor se ajuste a sus gustos e intereses, capacidad de inversión y familiaridad con el sector económico, sin embargo, los modelos de franquicia no son ejercicios empresariales eximidos de fracasos o reveses y no siempre es garantía de una inversión segura, en los anteriores apartados hemos explorado, a partir de la descripción de variables que operan el modelo de franquicias que se dedican a la prestación de servicios de formación extracurricular para niños, los riesgos que se detectan en el proceso de adaptación antes y después de la apertura de las unidades.

Los riesgos identificados, requieren mecanismos de mitigación que pueden ser implementados por el franquiciado o por el franquiciador, $y$, en la medida que se reconocen $y$ se atiendan mejoraran las posibilidades de éxito de la franquicia. En (Rigby \& Bilodeau, 2013), se establecieron 25 herramientas de gestión usadas más frecuentemente por gerentes y ejecutivos de empresas alrededor del mundo en sus procesos de gestión relacionadas con la planeación y la toma de decisiones.

En consecuencia, algunas de las herramientas que se citan en este documento relacionadas con la gestión y que pueden ayudar a la administración más acertada del riesgo se presentan en el siguiente diagrama 2 


\section{Diagrama 2 \\ Herramientas de gestión para mitigación de riesgos en las franquicias}

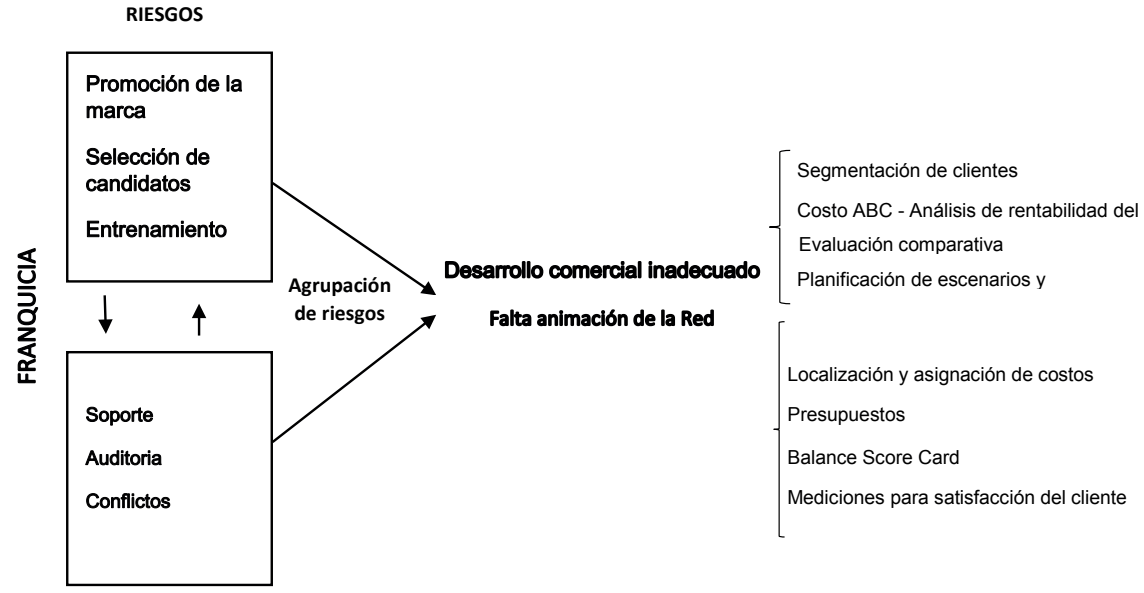

Fuente: Elaboración propia (2020)

La incorporación de las herramientas de gestión establecidas se presentan en el Cuadro 6 describiendo los beneficios que aportan y como mitigarían los riesgos asociados.

\section{Cuadro 6}

\section{Propósitos y beneficios de las herramientas de gestión en las franquicias}

\begin{tabular}{|c|c|c|c|}
\hline HERRAMIENTA & $\begin{array}{c}\text { PROPÓSITO DE LA } \\
\text { HERRAMIENTA }\end{array}$ & BENEFICIO FRANQUICIADO & BENEFICIO FRANQUICIANTE \\
\hline $\begin{array}{l}\text { La segmentación } \\
\text { de clientes }\end{array}$ & $\begin{array}{l}\text { "Es una herramienta para } \\
\text { conseguir mejoras. Supone } \\
\text { identificar grupos específi- } \\
\text { cos de clientes, con nece- } \\
\text { sidades y comportamientos } \\
\text { únicos, y definir las caracte- } \\
\text { rísticas demográficas rele- } \\
\text { vantes que los identifican." } \\
\text { (Best, 2007: 139) }\end{array}$ & $\begin{array}{l}\text { Conocimiento del mercado a ex- } \\
\text { plorar, incluso antes de la decisión } \\
\text { de comprar la franquicia, para re- } \\
\text { conocer intereses, gustos, hábitos } \\
\text { de compra de los clientes finales, } \\
\text { en el caso de estas franquicias } \\
\text { son los padres, y la disposición } \\
\text { de compra dependerá del diseño } \\
\text { de una propuesta de valor con el } \\
\text { servicio que proviene empaqueta- } \\
\text { do de la franquicia que responde a } \\
\text { sus gustos y cumplimiento de ex- } \\
\text { pectativas frente a la oportunidad, } \\
\text { tiempo, costo y beneficio. }\end{array}$ & $\begin{array}{l}\text { El mercado colombiano, no se com- } \\
\text { porta como los mercado locales de } \\
\text { donde provienen las franquicias, la } \\
\text { mayoría de estas marcas en Colom- } \\
\text { bia, vienen de países desarrollados, y } \\
\text { muchas han logrado una penetración } \\
\text { exitosa en México, Perú, Ecuador y } \\
\text { Chile, adaptándose a las diferencias } \\
\text { entre sus mercados de origen y el } \\
\text { mercado latinoamericano, y talvez } \\
\text { este éxito los ha inducido errónea- } \\
\text { mente a creer que la cultura latinoa- } \\
\text { mericana es igual o sus mercados } \\
\text { emergentes funcionan igual, lo cual } \\
\text { sorprende frente a las dificultades } \\
\text { encontradas en el desarrollo de las } \\
\text { unidades y en los largos periodos de } \\
\text { tiempo que han requerido las marcas. }\end{array}$ \\
\hline
\end{tabular}




\begin{tabular}{|c|c|c|c|}
\hline HERRAMIENTA & $\begin{array}{c}\text { PROPÓSITO DE LA } \\
\text { HERRAMIENTA }\end{array}$ & BENEFICIO FRANQUICIADO & BENEFICIO FRANQUICIANTE \\
\hline $\begin{array}{l}\text { Costo } \mathrm{ABC}-\text { Para } \\
\text { determinar la } \\
\text { rentabilidad del } \\
\text { cliente. }\end{array}$ & $\begin{array}{l}\text { La contabilidad tradicional } \\
\text { no permite identificar el } \\
\text { aporte de cada cliente en } \\
\text { la generación de utilida- } \\
\text { des, ni analizar los costos } \\
\text { asociados con satisfacer } \\
\text { sus demandas. Por esto } \\
\text { utilizar una herramienta de } \\
\text { coste enfocada en el cliente } \\
\text { ayuda a mejorar la toma de } \\
\text { decisiones. (Kaplan \& Coo- } \\
\text { per, 2003) }\end{array}$ & $\begin{array}{l}\text { Ayudará en la estrategia de precios } \\
\text { y evitará desgastes innecesarios } \\
\text { en segmentos o clientes que no } \\
\text { añade valor al negocio. }\end{array}$ & $\begin{array}{l}\text { Diseñar junto con el franquiciado pro- } \\
\text { puestas de valor rentables, con base } \\
\text { en las expectativas de los clientes y } \\
\text { la rentabilidad que se puede esperar } \\
\text { de estos. Estudiar escenarios para } \\
\text { determinar indicadores de gestión } \\
\text { en la generación de rentabilidad por } \\
\text { segmento. }\end{array}$ \\
\hline $\begin{array}{l}\text { Planificación de } \\
\text { escenarios. }\end{array}$ & $\begin{array}{l}\text { La planificación de esce- } \\
\text { narios se erige como una } \\
\text { herramienta estratégica ya } \\
\text { que le permite a la organi- } \\
\text { zación mantenerse infor- } \\
\text { mada sobre los cambios } \\
\text { que se susciten en materia } \\
\text { económica, política, social, } \\
\text { competitiva y tecnológica } \\
\text { que acontecen en el entor- } \\
\text { no y que tendrán impacto } \\
\text { en el futuro de largo o me- } \\
\text { diano plazo de la organi- } \\
\text { zación. } \\
\text { (Yori Conill, et al. } 2011 \text { : } \\
\text { 234) }\end{array}$ & $\begin{array}{l}\text { La realización de planes de es- } \\
\text { cenarios utilizando diversos mé- } \\
\text { todos, sumerge al franquiciado } \\
\text { en el contexto donde la marca se } \\
\text { desarrollará y le permite conocer } \\
\text { todos los factores que la afectan y } \\
\text { la viabilidad de unidades en uno u } \\
\text { otro entorno. }\end{array}$ & $\begin{array}{l}\text { Conocimiento macro y micro eco- } \\
\text { nómico, político, social, cultural del } \\
\text { lugar de adopción. Ideal para tomar } \\
\text { decisiones de cobertura e internacio- } \\
\text { nalización. }\end{array}$ \\
\hline $\begin{array}{l}\text { Evaluación com- } \\
\text { parativa }\end{array}$ & $\begin{array}{l}\text { Detectar fortalezas y bue- } \\
\text { nas prácticas en otros } \\
\text { sectores o incluso en los } \\
\text { competidores, es una al- } \\
\text { ternativa en la búsqueda } \\
\text { de soluciones que permitan } \\
\text { mejorar su posición compe- } \\
\text { titiva. } \\
\text { (Best, 2007) }\end{array}$ & $\begin{array}{l}\text { Superar aspectos débiles detecta- } \\
\text { dos en la gestión de la franquicia } \\
\text { que pueden minimizarse acogien- } \\
\text { do las mejoras que otros sectores } \\
\text { o competidores han alcanzado. }\end{array}$ & $\begin{array}{l}\text { Identificar valores de ventaja compe- } \\
\text { titividad que refuercen la promoción } \\
\text { de marca. }\end{array}$ \\
\hline $\begin{array}{l}\text { Mediciones para } \\
\text { satisfacción del } \\
\text { cliente }\end{array}$ & $\begin{array}{l}\text { Relaciones satisfactorias } \\
\text { con los clientes se traducen } \\
\text { en mayores niveles de ren- } \\
\text { tabilidad. (Best, 2007: 9) }\end{array}$ & $\begin{array}{l}\text { Identificar los elementos que los } \\
\text { clientes perciben como más signi- } \\
\text { ficativos y de esta manera dismi- } \\
\text { nuir inconformidades y retiro del } \\
\text { servicio. }\end{array}$ & $\begin{array}{l}\text { Consolidación de la marca, incremen- } \\
\text { to del posicionamiento y conocimien- } \\
\text { to de la marca. }\end{array}$ \\
\hline Costos & $\begin{array}{l}\text { Determinar la inversión ne- } \\
\text { cesaria para la prestación } \\
\text { del servicio. } \\
\text { (Horngren et al. 2006) }\end{array}$ & $\begin{array}{l}\text { Ayuda en la definición de precios } \\
\text { y establecimiento de margen de } \\
\text { rentabilidad que satisfaga los inte- } \\
\text { reses de la unidad. }\end{array}$ & $\begin{array}{l}\text { Establecimiento de indicadores de } \\
\text { operación mínimos y máximos entre } \\
\text { los cuales deben desenvolverse las } \\
\text { unidades. }\end{array}$ \\
\hline
\end{tabular}




\begin{tabular}{|c|c|c|c|}
\hline HERRAMIENTA & $\begin{array}{l}\text { PROPÓSITO DE LA } \\
\text { HERRAMIENTA }\end{array}$ & BENEFICIO FRANQUICIADO & BENEFICIO FRANQUICIANTE \\
\hline Presupuestos & $\begin{array}{l}\text { Instrumento eficaz para la } \\
\text { administración de los cos- } \\
\text { tos, útil para la planeación, } \\
\text { reducción y control de } \\
\text { estos. Además de una } \\
\text { medida para evaluar el } \\
\text { desempeño organizacional } \\
\text { (Horngren et al. 2006) }\end{array}$ & $\begin{array}{l}\text { Estimar y proyectar las inversio- } \\
\text { nes, los recursos y los límites de } \\
\text { gasto para hacer sostenible el fun- } \\
\text { cionamiento de la franquicia. }\end{array}$ & $\begin{array}{l}\text { Establecer indicadores de gestión } \\
\text { entre los cuales deban desenvolver- } \\
\text { se las unidades, de esta manera se } \\
\text { acompaña la gestión y se mantiene } \\
\text { equilibrio entre las franquicias que } \\
\text { conforman la red. }\end{array}$ \\
\hline $\begin{array}{l}\text { Balance Score } \\
\text { Card }\end{array}$ & $\begin{array}{l}\text { Sistema de medición del } \\
\text { desempeño realizando un } \\
\text { balance entre los indicado- } \\
\text { res financieros y no finan- } \\
\text { cieros, para identificar el } \\
\text { alcance de las metas orga- } \\
\text { nizacionales. } \\
\text { (Horngren et al. 2006) }\end{array}$ & $\begin{array}{l}\text { Gestionar el cumplimiento de las } \\
\text { metas trazadas. }\end{array}$ & $\begin{array}{l}\text { Una herramienta útil para el segui- } \\
\text { miento de las unidades y control de } \\
\text { los indicadores. }\end{array}$ \\
\hline
\end{tabular}

Fuente: Elaboración propia (2020)

\section{Conclusión}

Los procesos de adaptación de franquicias dedicadas a la formación extracurricular para niños en el país, plantean retos importantes para franquiciados y franquiciadores. Los hallazgos sobre los riesgos de adaptación identificados en las marcas, evidencian la necesidad de realizar esfuerzos adicionales para la comprensión de los mercados locales, lo que demanda un franquiciado con capacidades proactivas que de hábil lectura del entorno donde la unidad está inmersa y proponga mejoras pertinentes para afianzar más rápidamente la marca.

Aspectos positivos también se observan en la adaptación de franquicias, como la flexibilización de la casa matriz en la reconfiguración de la propuesta de operación original abandonando inversiones innecesarias, que permita en etapas tempranas de la apertura de unidades, una movilización de recursos hacia aspectos promocionales y de acercamiento de la marca a los públicos objetivos. Lo anterior también tiene un efecto positivo en la estructura de precios y facilita la competitividad de las unidades.

La capacitación constante, el acompañamiento y el entendimiento en todos los niveles de los franquiciados con la casa matriz son aspectos a considerar para incrementar la posibilidad de éxito de la franquicia.

El modelo conceptual de gestión incorporado en este documento es un punto de partida para estandarizar, como es necesario en este formato de negocio, estrategias que minimicen los riesgos identificados en la adaptación. Son herramientas que además pueden contribuir a la rápida toma de decisiones, establecimiento y medición de indicadores y alcance de los objetivos empresariales. 
Agudelo Cotes, Karen J.; Bedoya Gómez, Diana C.

Riesgos de adaptación en franquicias: herramientas de contabilidad de gestión para mitigarlos

\section{Referencias bibliográficas}

Asociación Española de Contabilidad y Administración de Empresas -AECA. (2008). La contabilidad de gestión en las empresas franquiciadoras. Madrid: AECA.

Best, R. (2007). Marketing Estratégico. Pearson Educación.

Cámara Colombiana de Franquicias. (viernes de junio de 2018). Durante el último año, el número total de franquicias ascendió a 506 en Colombia. La República.

Cordobés, M., \& Soldevila , P. (2015). Management Control in Interorganizational Relationships. The case of Franchises. Innovar Journal, 23-36. http://dx.doi.org/10.15446/ innovar.v25n58.52357

Eroglu, S. (1992). The Internationalization Process of Franchise Systems: A Conceptual Model. International Marketing Review, 19-30. https:// www.emerald.com/insight/content/ doi/10.1108/02651339210020277/ full/html

European Franchise Federation. (2018). European Franchise Report. European Franchise Federation. https://eff-franchise.com/

Feria Internacional de Negocios y Franquicias. (11 de 2019). FANYF. https://www.fanyf.com/directorio/

Horngren, C., Sundem, G., \& Stratton, W. (2006). Contabilidad Administrativa. Pearson Education.

International Franchise Association. (2019). Franchise Business Economic Outlook for 2020. Washington, DC: International Franchise Association.

Kaplan, R., \& Cooper, R. (2003). Coste y Efecto. Cómo usar el ABC, ABM y el $A B B$ para mejorar la gestión, los procesos y la rentabilidad. Ediciones
Gestión 2000.

Lanchimba, C., \& Medina, D. (2018). Impacto del franquiciamiento. Revista Problemas del Desarrollo, 95-118. https://bit.ly/3xIRNLf

Luangsuvimol, T., \& Kleiner, B. (2004). Effective Franchise Management. Management Research news, 6371. https://www.researchgate.net/ publication/235265390 Effective franchise management

Michael, S. C. (2014). Can franchising be an economic development strategy? An Empirical Investigation. Small Business Economic, 611620. https://www.researchgate. net/publication/263726499 Can franchising be an economic development strategy An empirical investigation

Oriol Salas, A., Falguera, J., Puig Pla, X., \& Soldevila, P. (2014). Aspectos que influyen en las diferencias internacionales en materia de Contabilidad de Gestión. Centro de Investigación en Economía Financiera y Contabilidad de la Universidad Pompeu Fabra.

Osorio Agudelo, J., Álvarez Arroyave, J., \& Atehortúa Castrillón, T. (2015). Caminos de Investigación a la Contabilidad de Gestión. Valencia.

Ramírez, C., Velez, M., \& Álvarez, M. (2013). Como controlan los franquiciadores españoles a sus franquiciados. Revista de Contabilidad, 1-10. https://doi. org/10.1016/S1138-4891(13)700013

Rigby, D., \& Bilodeau, B. (2013). Management tools and trends. Bain \& Company Inc.

Rosado Serrano, A., Paul, J., \& Dikova, D. (2018). International franchising: A literature review and research 
pp. 832-851

Revista Venezolana de Gerencia, Año 26 No. 95, 2021

agenda. Journal of Business Research, 238-257. https://doi. org/10.1016/j.jbusres.2017.12.049

Salgado Castillo, J. (2014). Tendencias en Contabilidad de gestión: una mirada a su evolución (finales del siglo xix y siglo xx). Cuadernos de Contabilidad, 787-805. https://dialnet.unirioja.es/ servlet/articulo?codigo $=5447029$

Shane, S. (1996). Why Franchise companies expand overseas. Journal of Business Venturing, 7388. $\quad$ https://doi.org/10.1016/08839026(95)00110-7

Shanne, S., \& Spell, C. (1998). Factors for New Franchise Success. MIT
Sloan Management Review. https:// sloanreview.mit.edu/article/factorsfor-new-franchise-success/

Wu, C.-W. (2015). Antecedents of franchise strategy and performance. Journal of business research, 1581$1588 . \quad$ https://doi.org/10.1016/j. jbusres.2015.01.055

Yori Conill, L., Hernandez de Velazco J., \& Chamaceiro Hernández, A. (2011). Planificación de escenarios: una herramienta estratégica para el análisis del entorno. Revista Venezolana de Gerencia, 274-290. https://produccioncientificaluz.org/ index.php/rvg/article/view/10617 